\title{
Predicting Peak Oxygen Uptake From the 6-Minute Walk Test in Patients With Pulmonary Hypertension
}

\author{
Augusto G. Zapico, PhD; Darío Fuentes, MS; Miguel A. Rojo-Tirado, PhD; Francisco J. Calderón, MD, PhD; \\ Erika B. Rosenzweig, MD; Robert P. Garofano, EdD
}

PURPOSE: To determine whether the 6-minute walk test (6MWT) is a predictor of peak oxygen uptake $\left(\dot{\mathrm{V}}_{2}\right)$ in patients with idiopathic pulmonary arterial hypertension (IPAH).

METHODS: A total of 157 patients treated for IPAH completed a 6MWT in accordance with American Thoracic Society standards. Heart rate, pulse oximetry, and Borg rating of perceived exertion were determined at baseline and posttest. A cardiopulmonary exercise test (CPET) was performed on a cycle ergometer using a ramp protocol. Participants were stratified post hoc by age into 3 groups: children $(n=26)$, adolescents $(\mathrm{n}=49)$, and adults $(\mathrm{n}=82)$. Multiple linear regression analysis was performed to predict peak $\dot{\mathrm{V}}_{2}$ from the 6MWT.

RESULTS: The regression equation generated for the children was the only model to strongly predict peak $\dot{\mathrm{V}}_{2}(r=0.87 ; P<.001)$. Similar models for adolescent $(r=0.59 ; P<.001)$ and adult groups $(r=0.68$; $P<.001)$ did not achieve the same level of correlation.

CONCLUSION: Six-Minute Walk Test was able to accurately predict peak $\dot{\mathrm{V}}_{2}$ in children (6-12 years) with IPAH; however, the model was weaker for predicting peak $\dot{\mathrm{V}}_{2}$ in older populations. These findings suggest that 6 MWT may be as valid a test for predicting peak $\dot{\mathrm{V}}_{2}$ in children with IPAH as CPET measured peak $\dot{\mathrm{V}}_{2}$. In older IPAH populations, CPET is the only reliable method for the evaluation of peak $\dot{\mathrm{V}}_{2}$.

\author{
$\begin{array}{lllllllllllllllll}K & E & Y & \text { W } & \mathrm{O} & \mathrm{R} & \mathrm{D} & \mathrm{S}\end{array}$ \\ 6-Minute Walk Test \\ exercise testing \\ oxygen uptake \\ pulmonary hypertension
}

Author Affiliations: Technical University of Madrid, Madrid, Spain (Drs Zapico and Calderón and Messrs Fuentes and Rojo-Tirado); Complutense University of Madrid, Madrid, Spain (Dr Zapico); and New York Presbyterian Hospital/Columbia University Medical Center, New York (Mr Fuentes and Drs Rosenzweig and Garofano).

Conflicts of Interest: no conflicts were declared.

Correspondence: Dario Fuentes, MS, PFT Laboratories, Pulmonary \& Critical Care Medicine, University of Michigan Health System, 1500 E Medical Center Dr, Ann Arbor, MI 48109 (fuentesd@umich.edu).

DOI: 10.1097/HCR.0000000000000174
The cardiopulmonary response to exercise is an important clinical assessment tool, as it provides an integrated assessment of the respiratory, cardiac, and metabolic systems. The current gold standard method for assessing aerobic exercise response in humans is the maximum incremental cardiopulmonary exercise test (CPET). Peak $\mathrm{VO}_{2}$, measured by a CPET, has been widely used to assess functional capacity and predict survival in patients with advanced symptomatic heart failure and respiratory dysfunction. ${ }^{1,2}$ However, CPET requires the use of expensive equipment and trained medical team supervision to conduct and evaluate each test.

Submaximal tests, like the 6-minute walk test (6MWT), provide useful information on daily physical performance that are safe, simple, standardized, and inexpensive. The 6MWT offers a better estimation of daily living activities than other walk tests ${ }^{3}$ and the American Thoracic Society has endorsed and published guidelines for performing the 6MWT in clinical settings. ${ }^{4}$ 
The 6MWT is an independent predictor of morbidity and mortality in adult patients with heart failure, ${ }^{1}$ left ventricular dysfunction, ${ }^{5}$ chronic respiratory disease,${ }^{6}$ cystic fibrosis, and pulmonary hypertension. ${ }^{2}$ In addition, the 6MWT is a significant predictor of survival in patients with pulmonary hypertension ${ }^{7-11}$ and is used in clinical trials for IPAH medications. ${ }^{12}$

In addition to other health limiting and debilitating conditions, patients with lung or heart disease may show a reduced 6-minute walk distance (6MWD). ${ }^{13-16}$ Greater pre-transplantation 6MWD also correlates with shorter intensive care unit stays after pediatric lung transplantation. ${ }^{17}$

Correlation between $\mathrm{VO}_{2}$ from CPET and 6MWT in healthy children ${ }^{18}$ and correlation between CPET and 6MWT in patients with $\mathrm{IPAH}^{2}$ has been reported by others but not prediction of $\mathrm{VO}_{2}$ peak from 6MWT. Ross et $\mathrm{al}^{19}$ attempted to predict peak $\mathrm{VO}_{2}$ from 6MWD in patients with different cardiopulmonary disorders, suggesting poor prediction accuracy for assessing individuals for clinical purposes. In an obese nonadult group, body mass index (BMI) and 6MWD were significant factors predicting peak $\mathrm{VO}_{2}$, although no coefficient of determination for the regression equation was cited. ${ }^{20}$ The 6MWT does not seem to predict peak $\mathrm{VO}_{2}$ in adult patients with heart failure. ${ }^{21}$ However, no other study has tried to predict peak $\mathrm{VO}_{2}$ from 6MWT in children, adolescents, or adults with IPAH. Maximal exercise testing in IPAH populations involves a certain risk and requires supervision by well-trained and experienced health care professionals.

Thus, the aim of this study was to determine whether the 6MWT could accurately predict peak $\mathrm{VO}_{2}$ in patients with IPAH, which could provide health care professionals an easily implemented and inexpensive tool in the evaluation and followup of patients.

\section{METHODS}

The study was conducted in accordance with the amended Declaration of Helsinki. The Columbia University Medical Center institutional review board approved the study protocol and waived the need for patient consent due to the retrospective nature of the study.

\section{Patients}

One hundred fifty-seven patients being treated for IPAH from 2003 to 2013 were included in this study. Patients were diagnosed with IPAH by right-sided heart catheterization measurement of arterial pulmonary pressures $>25 \mathrm{mmHg}^{22,23}$ Subjects were stratified post hoc by age into 3 groups (children: 6-12 years; adolescents: $13-18$ years; and adults: $\geq 19$ years) for between-group comparisons of anthropometric and 6MWT variables.

\section{Assessments}

Subjects were tested in the pediatric exercise laboratory as part of a standard of care for IPAH visits to the Pulmonary Hypertension Center of New York Presbyterian/Columbia University Medical Center. Each patient performed a pulmonary function test, CPET, and 6MWT, all on the same day. Height, weight, and age were gathered prior to the test. Patients were familiar with the tests.

\section{Pulmonary Function Test}

Forced vital capacity, slow vital capacity, and maximum voluntary ventilation were measured. Pulmonary function tests were performed on a Breeze Suite CPX Ultima (Medgraphics, St. Paul, MN).

\section{Cardiopulmonary Exercise Test}

The CPET was performed on a Lode Corival V2 (Lode BV, Groningen, the Netherlands) cycle ergometer following a ramp protocol with increments of 5, 10, 15, 20, or 25 watts/min (depending on fitness level and/ or previous tests) until volitional fatigue. Three minutes of baseline data were collected followed by 3 minutes of unloaded warm-up cycling. The ramp protocol was started after the 3-minute warm-up. Once the patient reached his or her maximum values, a 3-minute cooldown of unloaded cycling was performed as part of a 10-minute recovery period, until return to baseline levels and/or until symptoms, if any, resolved. ${ }^{24}$ The following data were collected during the cycle ergometer test: time, work in watts, real-time 12-lead electrocardiogram including heart rate using a CardioPerfect WorkStation (Welch Allyn, Skaneateles Falls, NY), systolic and diastolic blood pressures by using the standard arm cuff technique (W. A. Baum Company, Inc, Copiague, NY), noninvasive peripheral oxygen saturation using a Datex-Ohmeda 3900P pulse oximeter (Datex-Ohmeda, Madison, WI), $\mathrm{O}_{2}$ uptake and other gas exchange parameters through breath-bybreath analysis of expired air via Breeze Suite CPX Ultima (Medgraphics).

\section{MWT}

The 6MWT was performed in a 30-m hallway where the patients were instructed to walk as far as possible in 6 minutes by walking back and forth in a 30-m hallway at their own speed and pace following American Thoracic Society guidelines. ${ }^{4,25}$ Oxygen saturation using a finger sensor and a 3900P pulse oximeter (Datex-Ohmeda) and a rating of perceived 
exertion (RPE) using the Borg CR10 scale ${ }^{26}$ were collected at baseline and postwalk.

\section{Statistical Analysis}

The data were statistically analyzed using the PASW Statistics, version 18.0, for Windows (SPSS Inc, Chicago, IL). Continuous variables were presented as mean \pm standard deviation. To evaluate whether anthropometric and 6MWT variables were different between the 3 groups, analysis of variance (ANOVA) was used. Finally, 3 multiple linear regression models, one for each group of participants, were determined. Variables such as weight, height, BMI, sex, walk speed, resting heart rate, and RPE were included in the analyses. A backward elimination approach was used to finalize the regression models. If the slope for an independent variable was not found to be statistically significantly different than zero at $\alpha=.05$, that independent variable was excluded from the model. To assess the fit of the predictive model, we used conventional linear regression models according to the coefficient of determination $\left(R^{2}\right)$. A paired Student $t$ test was used to compare the mean values for the measured and the estimated peak $\mathrm{VO}_{2}$ measurements. Pearson correlation coefficient $(r)$ was used to assess the linear bivariate relationship among estimated and measured peak $\mathrm{VO}_{2}$. For all tests, a $P$ value $<.05$ was considered statistically significant.

\section{RESULTS}

The baseline characteristics of the sample are shown in Table 1. Among the 26 children, mean age was 9.96 \pm 1.66 years and $73 \%$ were female. In the group of adolescents $(\mathrm{n}=49)$, mean age was $15.33 \pm 1.80$ years and $47 \%$ were female. Among the 82 adults, mean age was $27.33 \pm 6.70$ years and $69.5 \%$ were female.

ANOVA revealed significant differences between age groups for height $\left(F_{2,156}=66.384 ; P<.001\right)$, weight $\left(F_{2,156}=24.453 ; P<.001\right)$, BMI $\left(F_{2,156}=11.943\right.$; $P<.001)$, and peak heart rate $\left(F_{2,156}=6.8 ; P=.001\right)$. No significant differences were found between groups for resting heart rate $\left(F_{2,156}=1.91 ; P=.152\right)$, 6MWD $\left(F_{2,156}=0.195 ; P=.823\right)$, walking speed $\left(F_{2,156}=\right.$ $0.195 ; P=.823)$, and $\operatorname{RPE}\left(F_{2,156}=1.39 ; P=.252\right)$.

For the linear regression analysis, the 3 age groups were children, adolescents, and adults and 3 independent multiple linear regressions were modeled. The variables - sex $(0=$ female, $1=$ male $)$; weight; height; BMI; walk speed; resting heart rate; and RPE-were included in the models. Final models included variables that yielded the highest $R^{2}$ values.

Table 2 shows the standardized and nonstandardized coefficients for the linear regression model. Using standardized coefficients, in the first model (children), weight, BMI, and height $(-4.72,3.10$, and 1.44 , respectively) contributed most to the model that predicted peak $\dot{\mathrm{V}}_{2}$. The remaining variables (sex, walk speed, RPE, and resting heart rate) contributed less to the prediction. In the second model (adolescents), the walk speed contributed most to the prediction model while RPE and height contributed less. In the third model (adults), the variable that contributed the most was 6MWD (0.48), before sex (0.30), height and weight to estimate peak $\dot{\mathrm{V}}_{2}$.

\section{Ta ble 1 Characteristics of Participants and 6MWT Data}

\begin{tabular}{|c|c|c|c|}
\hline & Children $(n=26)$ & Adolescents $(n=49)$ & Adults $(n=82)$ \\
\hline Age, y & $9.96 \pm 1.66$ & $15.33 \pm 1.80$ & $27.33 \pm 6.70$ \\
\hline Height, cm & $140.15 \pm 12.88$ & $160.98 \pm 9.48^{a}$ & $164.67 \pm 8.24^{\mathrm{a}, \mathrm{b}}$ \\
\hline Weight, kg & $35.09 \pm 13.31$ & $52.42 \pm 17.80^{\mathrm{a}}$ & $62.85 \pm 19.16^{\mathrm{a}, \mathrm{b}}$ \\
\hline $\mathrm{BMI}, \mathrm{kg} / \mathrm{m}^{2}$ & $17.37 \pm 3.81$ & $19.98 \pm 5.47$ & $23.02 \pm 5.96^{a, b}$ \\
\hline Resting HR, bpm & $90.19 \pm 22.01$ & $88.31 \pm 15.95$ & $83.84 \pm 15.51$ \\
\hline Peak HR, bpm & $153.12 \pm 26.22$ & $152.04 \pm 20.88$ & $137.10 \pm 28.58^{\mathrm{a}, \mathrm{b}}$ \\
\hline $6 \mathrm{MWD}, \mathrm{m}$ & $471.15 \pm 89.43$ & $455.78 \pm 84.63$ & $459.77 \pm 115.12$ \\
\hline Walking speed, $\mathrm{m} / \mathrm{s}$ & $1.31 \pm 0.25$ & $1.27 \pm 0.24$ & $1.28 \pm 0.32$ \\
\hline Dyspnea, Borg scale 0-10 & $1.60 \pm 2.11$ & $0.99 \pm 1.30$ & $1.31 \pm 1.44$ \\
\hline Peak $\dot{\mathrm{V}}_{2}, \mathrm{~mL} / \mathrm{kg} / \mathrm{min}$ & $22.07 \pm 6.67$ & $20.59 \pm 6.23$ & $16.03 \pm 6.33$ \\
\hline
\end{tabular}

Abbreviations: $\mathrm{BMI}$, body mass index; $\mathrm{HR}$, heart rate; $6 \mathrm{MWD}$, 6-minute walk distance; 6MWT, 6-minute walk test; $\dot{\mathrm{V}}_{2}$, oxygen uptake.

asignificantly different from children $(P<.05)$.

${ }^{b}$ Significantly different from adolescents $(P<.05)$. 


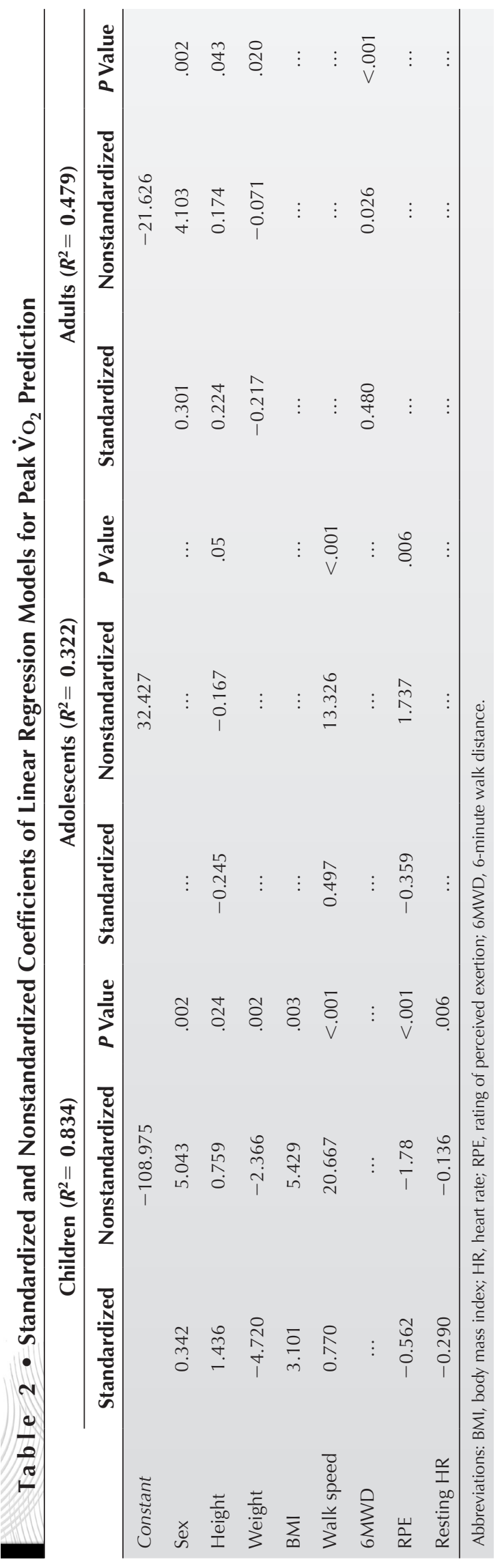

Assessment by the Student $t$ test showed no significant differences between the measured and the estimated peak $\dot{\mathrm{V}}_{2}$ for children, adolescents, and adults. There were significant correlations between measured and estimated peak $\dot{\mathrm{V}}_{2}$ in the 3 groups (children, $r^{2}=0.873$; adolescents, $r^{2}=0.591$; and adults, $r^{2}=0.681, P<.001$ for all). Figure 1 shows this correlation between measured peak $\dot{\mathrm{V}}_{2}$ from the exercise test (peak $\dot{\mathrm{V}}_{2}$-CPET) and estimated peak $\dot{\mathrm{V}} \mathrm{O}_{2}$ from 6MWT (peak $\dot{\mathrm{V}}_{2}-6 \mathrm{MWT}$ ) for all 3 groups (children, adolescents, and adults) expressed in $\mathrm{mL} / \mathrm{kg} / \mathrm{min}$.

\section{DISCUSSION}

The primary result of this study was that a simple 6MWT may be able to predict peak $\dot{\mathrm{VO}}_{2}$ in children with IPAH. However, the 6MWT was not able to predict peak $\dot{\mathrm{VO}}_{2}$ in other patients with IPAH. This is the first study that attempted to predict peak $\dot{\mathrm{V}}_{2}$ from 6MWT solely in patients with IPAH. Being able to predict peak $\dot{\mathrm{V}}_{2}$ from 6MWT would give physicians and other health care professionals an easy, inexpensive, and simple tool for the followup of the disease. The 6MWT was a strong predictor of peak $\dot{\mathrm{VO}}_{2}$ in children; however, the predictions were weaker in adolescents or adults.

Although peak $\dot{\mathrm{VO}}_{2}$ was predicted $\left(r^{2}=0.83\right)$ in the children's group, peak $\dot{\mathrm{V}}_{2}$ alone does not provide enough information for the treatment and prognosis of patients with IPAH. Peak $\dot{\mathrm{V}}_{2}$ is only 1 of the many variables obtained from a CPET. Peak $\dot{\mathrm{V}}_{2}$ would only show a decrease in aerobic fitness in these patients but it would not determine what contributed to the decrease. In children, up to 12 years of age, the 6MWT may be used to predict peak $\dot{\mathrm{V}}_{2}$, although a previous CPET would always be highly recommended and the 6MWT could be used as a followup assessment until adolescence.

Correlation between $\dot{\mathrm{VO}}_{2}$ from CPET and 6MWT in healthy children ${ }^{18}$ and correlation between CPET and 6MWT in patients with IPAH ${ }^{2}$ have been reported by others, but no correlation between 6MWT predicted $\dot{\mathrm{V}}_{2}$ and measured $\dot{\mathrm{V}}_{2}$ has been reported. However, Ross et $\mathrm{al}^{19}$ attempted to predict peak $\mathrm{V}_{2}$ from 6MWD in patients with different cardiopulmonary disorders and reported poor prediction accuracy for assessing individuals for clinical purposes. Body mass index and 6MWD were the only significant factors to predict peak $\dot{\mathrm{V}}_{2}$, in obese nonadult patients, although no coefficient of determination for the regression equation was reported. ${ }^{20}$ 6MWT does not appear to accurately predict peak $\dot{\mathrm{V}}_{2}$ in adult patients with heart failure. ${ }^{21}$ 
A
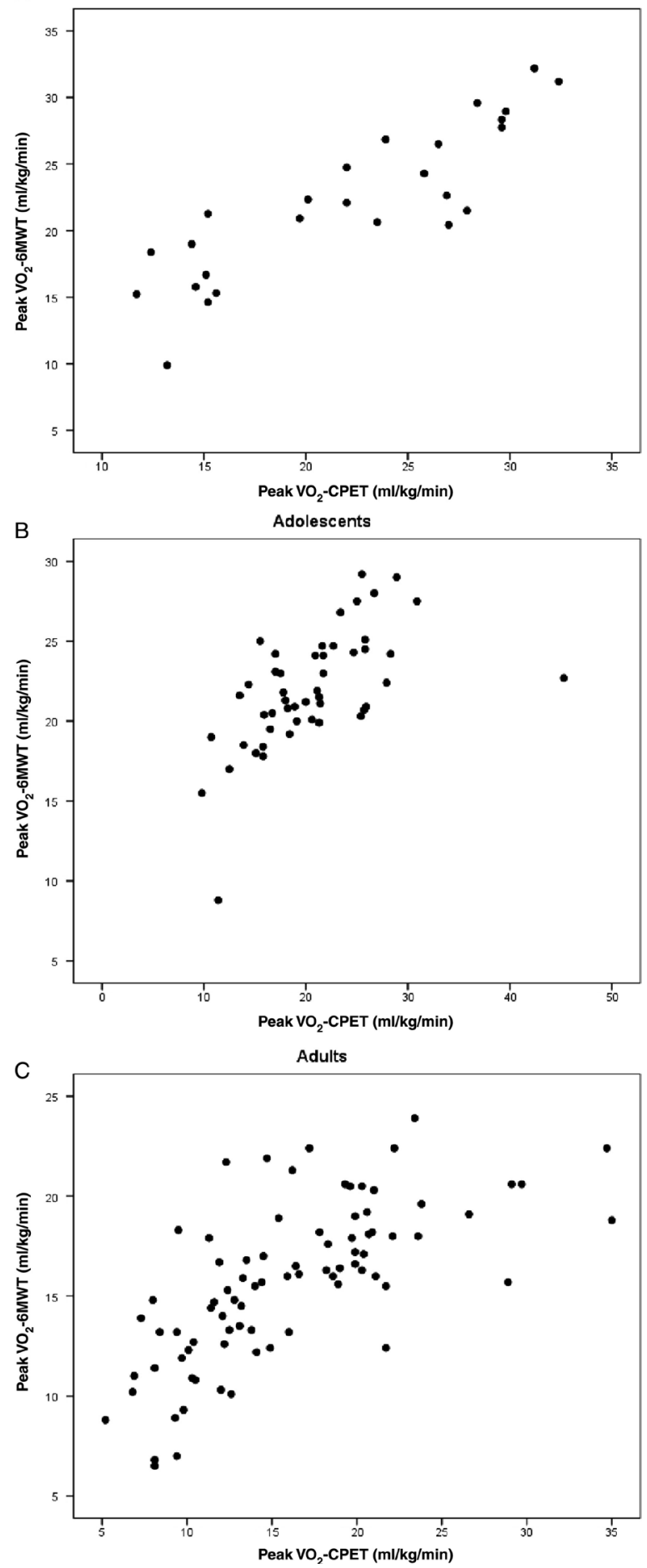

Figure 1. Measured (CPET) versus predicted (6MWT) values for peak $\dot{\mathrm{V}}_{2}$ in (A) children, (B) adolescents, and (C) adults. CPET indicates cardiopulmonary exercise test; $\dot{\mathrm{V}}_{2}$, oxygen uptake; $6 \mathrm{MWT}$, 6-minute walk test.

However, no other study has tried to predict peak $\dot{\mathrm{V}}_{2}$ from 6MWT in children, adolescents, or adults with IPAH.
Weight, BMI, and height have been cited as the most important variables affecting 6MWD. ${ }^{27,28}$ Consistent with these findings, our models showed that these 3 variables have the strongest effect on the linear regression to predict peak $\mathrm{V}_{2}$ in children.

As expected, IPAH subjects showed lower 6MWD than healthy patients from other studies: children with IPAH averaged $471.15 \pm 89.43$ meters versus $664 \pm$ 65.3 meters $^{29}$ and $619.2 \pm 115.2$ meters $^{30}$ in healthy children; adolescents with IPAH walked $455.78 \pm$ 84.63 meters versus $699.2 \pm 87$ meters $^{30}$ found in healthy adolescents; IPAH adults averaged a 6MWD of $459.77 \pm 115.12$ meters versus the distances averaged by other healthy adults of $615.5 \pm 44$ meters $^{31}$ and 672 \pm 83 meters. $^{32}$

Although a 6MWT may not be robust enough to predict peak $\dot{\mathrm{V}}_{2}$ in adolescents and adults, it remains a very important tool to predict morbidity and mortality in a clinical setting. It is also important in predicting shorter intensive care unit stays after pediatric lung transplantation, ${ }^{17}$ as well as predicting morbidity and mortality in adult patients with left ventricular dysfunction. ${ }^{5}$ The 6MWT is a significant predictor of survival in patients with IPAH. ${ }^{7-11}$ It is still used in clinical trials for IPAH medication use. ${ }^{12}$

Although performing a simpler submaximal test, like the 6MWT, with children with IPAH may predict peak $\dot{\mathrm{V}}_{2}$, it would not give health care professionals the necessary information to evaluate functional capacity. The 6MWT is not able to predict peak $\dot{\mathrm{V}}_{2}$ in most patients with pulmonary hypertension, but it is an important tool for the evaluation and followup of patients with IPAH.

The small sample size, specifically in the children's group ( $\mathrm{n}=26$, with only 7 males), is a limitation of this study. In future research, a larger sample size, especially in the children's group, is needed. The small sample size in this study prevented us from validating the regression equations. Also, future studies comparing each group with a control group could shed more light on how and what prediction equations used in the study would apply to a normal or healthy population.

\section{CONCLUSION}

The measured peak $\dot{\mathrm{V}}_{2}$ of the patients varied greatly by age. A linear regression equation developed from the 6MWT was able to accurately predict peak $\dot{\mathrm{VO}}_{2}$ in children (6-12 years) with IPAH; however, the model failed to predict peak $\dot{\mathrm{VO}}_{2}$ in older populations. These findings suggest that peak $\mathrm{V}_{2}$ measured by CPET is still the gold standard for assessing aerobic exercise capacity in patients with IPAH. However, the 6MWT 
could still be a valuable test for prognostic purposes when performing a CPET is not an option. It could also be of great value as a complement to the CPET. Future research should be conducted to validate the regression equations.

\section{References}

1. Cahalin LP, Mathier MA, Semigran MJ, Dec GW, DiSalvo TG. The Six-Minute Walk Test predicts peak oxygen uptake and survival in patients with advanced heart failure. Chest. 1996; 110(2):325-332.

2. Oudiz RJ, Barst RJ, Hansen JE, et al. Cardiopulmonary exercise testing and six-minute walk correlations in pulmonary arterial hypertension. Am J Cardiol. 2006;97(1):123-126.

3. Solway S, Brooks D, Lacasse Y, Thomas S. A qualitative systematic overview of the measurement properties of functional walk tests used in the cardiorespiratory domain. Chest. 2001;119(1): 256-270.

4. American Thoracic Society. ATS statement: guidelines for the SixMinute Walk Test. Am J Respir Crit Care Med. 2002;166(1):111-117.

5. Bittner V, Weiner DH, Yusuf S, et al. Prediction of mortality and morbidity with a 6-Minute Walk Test in patients with left ventricular dysfunction. SOLVD Investigators. JAMA. 1993;270(14): 1702-1707.

6. Ashutosh K, Dunsky M. Noninvasive tests for responsiveness of pulmonary hypertension to oxygen. Prediction of survival in patients with chronic obstructive lung disease and cor pulmonale. Chest. 1987;92(3):393-399.

7. Groepenhoff H, Vonk-Noordegraaf A, van de Veerdonk MC, Boonstra A, Westerhof N, Bogaard HJ. Prognostic relevance of changes in exercise test variables in pulmonary arterial hypertension. PLoS One. 2013;8(9):e72013.

8. Benza RL, Miller DP, Gomberg-Maitland M, et al. Predicting survival in pulmonary arterial hypertension: insights from the Registry to Evaluate Early and Long-Term Pulmonary Arterial Hypertension Disease Management (REVEAL). Circulation. 2010;122(2):164-172.

9. Miyamoto S, Nagaya N, Satoh T, et al. Clinical correlates and prognostic significance of Six-Minute Walk Test in patients with primary pulmonary hypertension. Comparison with cardiopulmonary exercise testing. Am J Respir Crit Care Med. 2000;161 (2 Pt 1):487-492.

10. Paciocco G, Martinez FJ, Bossone E, Pielsticker E, Gillespie B, Rubenfire M. Oxygen desaturation on the Six-Minute Walk Test and mortality in untreated primary pulmonary hypertension. Eur Respir J. 2001;17(4):647-652.

11. Timofte IL, Tonelli A, Akindipe O, Baz M. SIx-Minute Walk Test and mortality in patients with advanced lung disease. Chest. 2010;138(4_MeetingAbstracts):544A-544A.

12. Mathai SC, Puhan MA, Lam D, Wise RA. The minimal important difference in the 6-Minute Walk Test for patients with pulmonary arterial hypertension. Am J Respir Crit Care Med. 2012; 186(5):428-433.

13. Alameri HF, Sanai FM, Al Dukhayil M, et al. Six Minute Walk Test to assess functional capacity in chronic liver disease patients. World J Gastroenterol. 2007;13(29):3996-4001.
14. Hassan J, van der Net J, Helders PJ, Prakken BJ, Takken T. Six-Minute Walk Test in children with chronic conditions. $\mathrm{BrJ}$ Sports Med. 2008;44(4):270-274.

15. Morinder G, Mattsson E, Sollander C, Marcus C, Larsson UE. Six-Minute Walk Test in obese children and adolescents: reproducibility and validity. Physiother Res Int. 2009;14(2): 91-104.

16. Ruden E, Reardon DA, Coan AD, et al. Exercise behavior, functional capacity, and survival in adults with malignant recurrent glioma. J Clin Oncol. 2011;29(21):2918-2923.

17. Yimlamai D, Freiberger DA, Gould A, Zhou J, Boyer D. Pretransplant Six-Minute Walk Test predicts peri- and postoperative outcomes after pediatric lung transplantation. Pediatr Transplant. 2013;17(1):34-40.

18. Limsuwan A, Wongwandee R, Khowsathit P. Correlation between 6-Min Walk Test and exercise stress test in healthy children. Acta Paediatr. 2010;99(3):438-441.

19. Ross R, Murthy J, Wollak I, Jackson A. The Six Minute Walk Test accurately estimates mean peak oxygen uptake. BMC Pulm Med. 2010;10(1):31.

20. Vanhelst J, Vanhelst J, Fardy PS, Salleron J, Beghin L. The SixMinute Walk Test in obese youth: reproducibility, validity, and prediction equation to assess aerobic power. Disabil Rehabil. 2013;35(6):479-482.

21. Maldonado-Martin S, Brubaker PH, Kaminsky LA, Moore JB, Stewart KP, Kitzman DW. The relationship of a 6-min walk to $\dot{\mathrm{V}}_{2}$ and VT in older heart failure patients. Med Sci Sports Exerc. 2006;38(6):1047-1053.

22. Badesch DB, Champion HC, Gomez Sanchez MA, et al. Diagnosis and assessment of pulmonary arterial hypertension. J Am Coll Cardiol. 2009;54(1) (suppl):S55-S66.

23. Hoeper MM. The new definition of pulmonary hypertension. Eur Respir J. 2009;34(4):790-791.

24. American College of Sports Medicine. ACSM's Guidelines for Exercise Testing and Prescription. 7th ed. Philadelphia, PA: Lippincott Williams \& Wilkins; 2006.

25. Li AM, Yin J, Au JT, et al. Standard reference for the Six-MinuteWalk Test in healthy children aged 7 to 16 years. Am J Respir Crit Care Med. 2007;176(2):174-180.

26. Borg G. Borg's Perceived Exertion and Pain Scales. Champaign, IL: Human Kinetics; 1998.

27. Enright PL, Sherrill DL. Reference equations for the six-minute walk in healthy adults. Am J Respir Crit Care Med. 1998;158(5 Pt 1):1384-1387.

28. Enright PL. The Six-Minute Walk Test. Respir Care. 2003;48(8): 783-785.

29. Li AM, Yin J, Au JT, et al. Standard reference for the Six-Minute Walk Test in healthy children aged 7 to 16 years. Am J Respir Crit Care Med. 2007;176(2):174-180.

30. Geiger R, Strasak A, Treml B, et al. Six-Minute Walk Test in children and adolescents. J Pediatr. 2007;150(4):395-399. e392.

31. Chetta A, Zanini A, Pisi G, et al. Reference values for the 6-min walk test in healthy subjects 20-50 years old. Respir Med. 2006;100(9):1573-1578

32. Ozasa N, Morimoto T, Furukawa Y, Hamazaki H, Kita T, Kimura T. Six-minute walk distance in healthy Japanese adults. Gen Med. 2010;11(1):25-30. 